\title{
Comparative mapping and discovery of segregation distortion and linkage disequilibrium across the known fragrance chromosomal regions in a rice F2 population
}

\begin{abstract}
Segregation distortion analysis in plant breeding is highly important because deviation factors are occasionally serious barriers for introgression of genes of interest into cultivated species. Also, the identification of major QTLs by high linkage disequilibrium is highly useful for breeders in order for crop improvement programs and the further fine mapping of particular genes. Consequently, simultaneous consideration of segregation distortion and linkage disequilibrium leads to developing fertile hybrids and normal inheritance of agronomically favorable genes. Hence, the goal of this study was to investigate genomic regions known to contain fragrance quantitative trait loci (QTLs) in terms of segregation distortion and linkage disequilibrium pattern, and to seek for a possible relationship between them using codominant markers on chromosomes 3, 4, and 8 in a set containing 189 F2 progenies. For the first time, we discovered the presence and extent of segregation distortion and linkage disequilibrium across the fragrance chromosomal areas of rice. The high frequency of skewed allelic segregations in the chromosomes 3 and 4 supports reports of possible gametic selection (ga) and hybrid sterility (S) genes. Finally, the highest linkage disequilibrium values, the lowest recombination frequency, and the shortest distances were observed in the chromosomal regions without segregation distortion. High linkage disequilibrium correlations between markers at short distances may show that such markers can maintain high linkage disequilibrium with linked QTLs and are influential for both MAS and QTL mapping analysis. Moreover, an indirect relationship between segregation distortion and linkage disequilibrium was detected, and thus linkage disequilibrium maps were introduced as useful diagnostic tools for segregation distortion.
\end{abstract}

Keyword: Aromatic rice; Segregation distortion; Linkage disequilibrium; Microsatellite markers; Genetic map; Comparative map 